\title{
EFFECTS OF PHOSPHORUS DEFICIENCY ON THE ROOT GROWTH OF LENTIL SEEDLINGS (LENS CULINARIS MEDIK) GROWN IN RHIZOBOX
}

\author{
Bimal Chandra SARKer ${ }^{1}$ AND J. L KARMOKer \\ Department of Botany, University of Dhaka, Dhaka-1000, Bangladesh
}

Key words: Phosphorus deficiency, Root length, Root growth, Rhizobox, Lentil

\begin{abstract}
Phosphorus deficiency resulted in an increase in the length of primary root, length and number of lateral roots, root hairs and root meristem volume of the seedlings of lentil grown in rhizobox .
\end{abstract}

Absorption of nutrients of low mobility in soil, such as phosphorus, is strongly influenced by morphological characteristics of roots. Morphological responses of plants to phosphorus deficiency include enhanced root elongation (Raghothama 1999, Barber 1984), root hair formation (Ma et al. 2001, Marschner 1995), and decrease in root radius (Fohse et al.1991). Significant increase in the length of primary and secondary roots was observed in Horse gram (Microtyloma uniflorum Lam.), sesame (Sesamum indicum L.) and pearl millet (Pennisetum glaucumL.) plants grown in P-depleted soil (Anuradha and Narayanan 1997). Phosphorus deficiency caused a rapid elongation of the root at the initial stage and the stimulation of elongation was sustained even after transfer of plants to full nutrient medium (Ciereszko et al. 2002).

In this paper, the effect of phosphorus deficiency on the root length and number, and root hair length and number and overall root growth are reported.

Seeds of lentil (Lens culinaris Medik var. Barimasur-4) were collected through the courtesy of Bangladesh Agricultural Research Institute, Joydebpur, Gazipur, Bangladesh.

Plants were grown in rhizobox to study the root growth. A special nutrient solution developed in the laboratory of Institute of Plant Nutrition, University of Hohenheim, Germany was used. Phosphorus containing solution $(+\mathrm{P})$ was used as control and phosphorus free (-P) solution was used as phosphorus deficiency treatment.

Rhizobox used in this experiment was an wooden box having length $\times$ breadth $\times$ depth of 25 $\times 17 \times 5 \mathrm{~cm}$. Lower portion of the rhizobox was perforated for drainage of excess solution. The wooden box was fitted with a transparent plastic lid so that the elongation of root can be observed and measured. There was a slit in front of the box to facilitate the growth of the stem. The rhizobox was filled with $2.5 \mathrm{~kg}$ of purified quartz sand. Some sterilized seeds were germinated on moist filter paper placed in petri dishes at $25 \pm 1^{\circ} \mathrm{C}$. The sprouting was considered as the zero age of the seedlings. A one-day-old seedling was placed in the rhizobox filled with quartz sand where the radicle was in the sand, while the plumule was protruding outside through the hole in the ryhizobox. The sand of three rhizoboxes with seedlings was moistened with $+\mathrm{P}$ solution which was useds as control and that of other three rhizoboxes were moistened with -P solution. The nutrient was supplied every day. The rhizoboxes were kept in a net house at the Department of Botany, in a natural environmental condition.

A transparent sheet was cut having the same size as the plastic lid. The sheet was placed on the lid of the rhizobox. The roots of the seedling in the rhizobox was traced on the transparent sheet. This was done at 2, 4, 6 and 8-days of germination. The length of the traced root was measured and recorded. The rate of growth of roots from 2 to 8 -days of germination was calculated from the data collected from the length of the root traced in the transparent paper.

\footnotetext{
${ }^{1}$ Department of Botany, Ananda Mohan College, Mymensingh, Bangladesh.
} 
For measurement of length and number of root hairs, eye-piece graticule was placed on transparent lid of rhizobox along the root where root hair zone was situated. Root hairs were counted through eye-poiece graticule at $\times 10$ magnification within $1 \mathrm{~cm}$ root hair zone section following 2, 4, 6 and 8-days of phophorus deficiency.

Volume of primary root meristem was calculated using the formula suggested by Rost and Baum (1988). Three replicates were used for all observations and in each cause standard error of mean was calculated.

Phosphorus deficiency caused an increase in the primary root length of lentil from 7 to $18 \%$ at 2- to 8-day of treatment (Fig. 1a). The total lateral root length of lentil was increased from 7.9 to $68.5 \%$ at 2 to 8 -day of phosphorus deficiency treatment (Fig. 1b). Similarly, the total root length of lentil was increased from 30.7 to $80.6 \%$ from 2 to 8 -day of treatment following phosphorus deficiency treatment (Fig. 1c). Phosphorus deficiency resulted in an increase in the number of lateral roots from 20 to $45 \%$ from 2 to 4 -day and 70 to $67 \%$ from 6 to 8 -day of treatment (Fig. 1d). Under phosphorus deficiency, the total root length and number of lateral roots of the tolerant rice cultivars was increased more than that of the sensitive cultivars (Li et al. 2004).
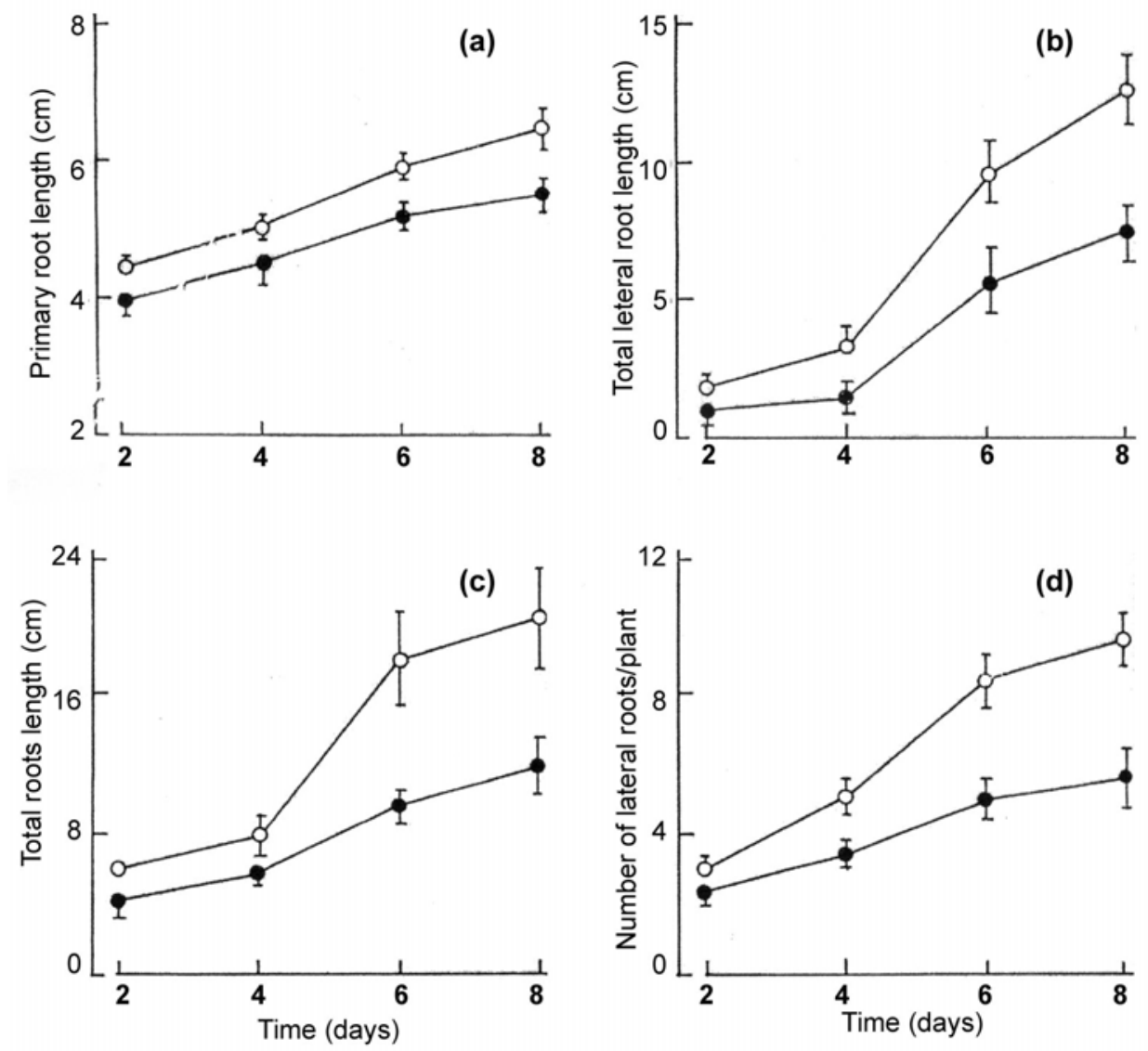

Fig. 1. Effects of phosphorus deficiency on the primary root length (a), total leteral roots length (b), total roots length (c) and number of lateral roots (d) of 8-day-old intact lentil seedling grown in rhizobox. Each value is the mean of three replicates. $\mathrm{n}=3$, vertical bars indicate standard error. Symbols: $-\bullet-=$ control, ${ }_{\mathrm{O}}-\mathrm{P}$-deficient. 
Low phosphate concentration of enhanced lateral root growth over primary root growth as indicated by increased lateral root number and length (Williamson et al. 2001). Phosphorus deficiency increased the root meristem volume of lentil from $24-46 \%$ at 2 to 8 -day of treatment (Fig. 2a). Ma and Liang (2004) observed that increased root meristem volume was in agreement with the increased root length of the phosphorus deficient plant. Phosphorus deficiency caused a 2- to 2.4-fold increase in the length of root hair in lentil from 6 to 8-day of treatment (Fig. 2b). Number of root hairs in P-deficient lentil was increased by 23.6 to $40.9 \%$ from 4 to 8 -day of treatment (Fig. 2c). Zhu et al. (2005) observed that in maize, the number of root hairs and root length was increased following P-deficiency treatment. Fohse et al. (1991) reported that the length and number of root hairs was increased in P-deficient plants.
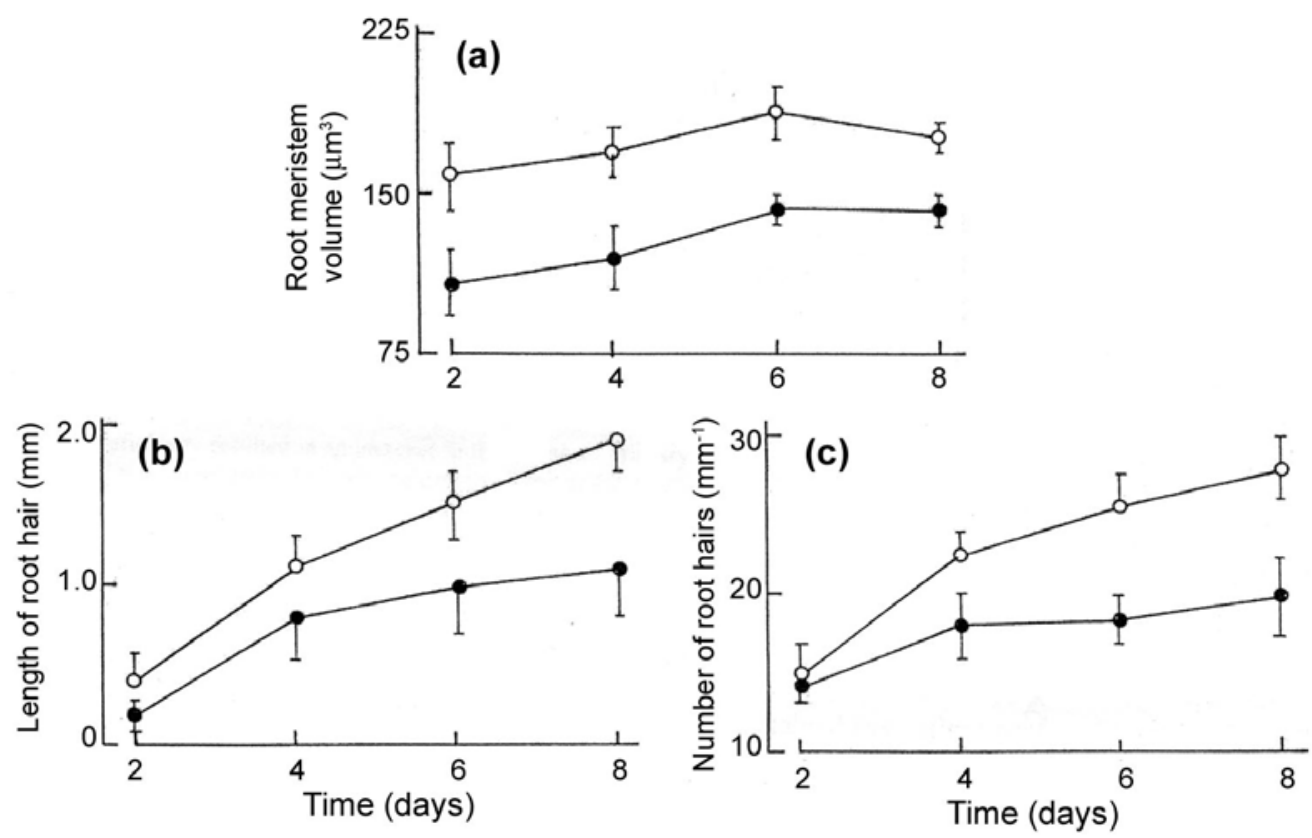

Fig. 2. Effects of phosphorus deficiency on the (a) root meristem volume, (b) length of root hair and (c) number of root hairs of 8-day-old intact lentil seedling grown in rhizobox. Otherwise as in Fig. 1.

The increase in the total root length and number of root hairs resulted in an increase in root surface area under phosphorus deficient condition. An increase in the root surface area in phosphorus -deficient plant may be considered as a strategy for enhancing phosphorus acquisition from P-deficient soil.

\section{References}

Anuradha M. and A. Narayanan. 1997. Root elongation of plants grown in phosphorus-deficient soil and vermaculite. Indian J. Plant Physiol. 2: 65-67.

Barber S.A. 1984. Soil nutrient bioavailability. A mechanistic approach. John Wiley \& Sons Inc, New York. $398 \mathrm{pp}$.

Ciereszko I., A. Janonis and M. Kociakowska. 2002. Growth and metabolism of cucuber in phosphatedeficient conditions. J. Plant Nutr. 25: 1115-1127. 
Fohse D., N. Classen and A. Jungk. 1991. Phosphorus efficiency of plants. II. Significance of root radius, root hairs and cation-anion balance for phosphorus influx in seven plant species. Plant Soil. 132: 261-272.

Li F., X.H. Pan, S.Y. Liu, M.Y. Li and F.S. Yang. 2004. Effects of phosphorus deficiency stress on root morphology and nutrient absorption of rice cultivars. Acta-Agronomica-Sinica 30: 538-442.

Ma X. and X. Liang. 2004. Research advances in mechanism of high phosphorus use efficiency of plants. Yingyong-Shengtai-Xuebao 15: 712-716.

Ma Z., D.G. Bielenbery, K.M. Brown and J.P. Lynch. 2001. Regulation of root hair density by phosphorus availability in Arabidopsis thaliana. Plant Cell Environ. 24: 459-467.

Marschner H. 1995. Mineral nutrition of higher Plants. 2nd edition, Academic Press, London. pp. 889.

Raghothama K.G. 1999. Phosphate acquisition. Ann. Rev. Plant Physiol. Plant Mol. Biol. 50: 665-693.

Rost T.L. and S. Baum. 1988. On the correlation of root length, meristem size and protoxylem tracheary element position in pea seedlings. Am. J. Bot. 75: 414-424.

Williamson C., P.C.P. Sebastein, F.H. Alastair and H.M.O. Leyer. 2001. Phosphate availability regulates root system architecture in Arabidopsis. Plant Physiol. 126: 875-882

Zhu J., S.M. Kaeppler and J.P. Lynch. 2005. Mapping of QTL controlling root hair length in maize (Zea mays L.) under phosphorus deficiency. Plant and Soil. 270: 299-310.

(Manuscript received on 9 June, 2009; revised on 12 December, 2009) 